$\underline{\text { Short communication }}$

\title{
Preparation and characterization of poly(L-phenylalanine) chiral stationary phases with varying peptide length
}

\begin{abstract}
Kaname Ohyama ${ }^{a}$, Kana Oyamada ${ }^{a}$, Naoya Kishikawa ${ }^{a}$, Yoshihito Ohba ${ }^{a}$, Mitsuhiro Wada ${ }^{\mathrm{b}}$, Toshihide Maki ${ }^{\mathrm{c}}$, Kenichiro Nakashima ${ }^{\mathrm{b}}$, Naotaka Kuroda ${ }^{\mathrm{a} *}$
\end{abstract}

${ }^{a}$ Department of Environmental and Pharmaceutical Sciences, Course of Pharmaceutical Sciences, Graduate School of Biomedical Sciences, Nagasaki University

${ }^{\mathrm{b}}$ Department of Clinical Pharmacy, Graduate School of Biomedical Sciences, Nagasaki University

${ }^{\mathrm{c}}$ Joint Research Center, Nagasaki University

* Corresponding author. Course of Pharmaceutical Sciences, Graduate School of Biomedical Sciences, Nagasaki University, Nagasaki, Japan. Tel.: +81-95-819-2894; fax: +81-95-819-2444. E-mail address: n-kuro@ nagasaki-u.ac.jp

Key words: L-phenylalanine / chiral stationary phase / peptide length / secondary structure / liquid chromatography 


\begin{abstract}
Three new chiral stationary phases with different lengths of L-phenylalanine peptide were prepared by solid-phase synthesis with Boc-L-phenylalanine on silica. The effect of phenylalanine peptide length on enantioselectivity was studied. The best separation of $R / S$-warfarin was achieved by the chiral stationary phase with intermediate peptide length. These stationary phases were found to exist mainly in $\alpha$-helical conformation by using FT-IR spectra. The end-capping reagents for the $\mathrm{N}$-terminus of the peptide were also evaluated.
\end{abstract}




\section{Introduction}

Enantiomers of a chirally active drug often have a dramatically different pharmacological or toxicological effect; therefore, the separation of enantiomers is very important. High performance liquid chromatography (HPLC) with a chiral stationary phase (CSP) is a popular method in the field of chiral separation because of its good reproducibility and preparative capability. The design and development of CSPs has attracted much attention for a number of years. To date, CSPs with several chiral selectors, such as cyclodextrins [1], macrocyclic antibiotics [2], polysaccharide derivatives [3], modified amino acid [4, 5], protein [6] and cinchona alkaloids [7], have been reported and some of them are commercially available.

Since CSPs with a protein chiral selector have excellent enantioselectivity, a peptide chiral selector would seem to offer promise. Indeed, research on peptide chiral selectors has progressed [8-14]. Li et al. developed polyproline-based CSPs and demonstrated their broad enantioselectivity in normal-phase mode [10, 11]. Also, Chen used poly( $N$-benzyl-L-glutamine)-coated silica for the chiral separation of hydantoins [13].

In this study, we developed a novel CSP with phenylalanine (Phe) peptide as chiral selector. The CSP was prepared on aminopropyl silica (APS) by solid-phase synthesis with Boc-L-Phe. For a peptide chiral selector, the secondary structure introduces more conformational rigidity into the selector and can influence its enantioselectivity. The secondary structure of a peptide varies by its length [15], thus in this study three CSPs with different Phe peptide lengths, $\mathrm{Phe}_{4}, \mathrm{Phe}_{8}$ and $\mathrm{Phe}_{12}$, were individually prepared. We studied the effect of peptide length on chiral separation in HPLC and also the effect of end-capping reagents for the $\mathrm{N}$-terminus. 


\section{Experimental}

\section{Chemicals}

APS (particle size, $5 \mu \mathrm{m}$; pore size, $120 \AA$; lot 1 and 2) was a kind gift from Daiso Chemical (Osaka, Japan). Boc-L-Phe was purchased from Peptide Institute (Osaka, Japan). Dicyclohexylcarbodiimide (DCC), $N, N$ '-diisopropylethylamine (DIEA), benzoyl chloride (BZ), triethylamine (TEA), methanol and ninhydrine were obtained from Wako Pure Chemicals (Osaka, Japan). 3,5-dinitrobenzoyl chloride (DNB), cyclohexanecarbonyl chloride $(\mathrm{CH})$ and trifluoroacetic acid (TFA) were from Tokyo Chemical Industry (Tokyo, Japan). Dichloromethane (DCM), ethanol, sodium perchlorate, perchloric acid were from Kishida Chemicals (Osaka, Japan). HPLC grade of acetonitrile, trihexyphenidyl hydrochloride, ketoprofen, chloropheniramine maleate and benzoin were purchased from Wako. Warfarin was obtained from Sigma (St. Louis, USA). Optically pure warfarin was prepared from racemic warfarin, using a fractional crystallization method [16].

Preparation of APS-(Phe $)_{n}$-BZ by solid-phase synthesis

Boc-L-Phe (1.5 g), DCC (1.23 g) and DIEA (100 $\mu \mathrm{l})$ were added to a suspension of APS (lot 1, $0.7 \mathrm{mmol} / \mathrm{g}, 5 \mathrm{~g})$ and DCM $(25 \mathrm{ml})$ and the mixture was stirred at room temperature for $2 \mathrm{~h}$. After filtration and washing with DCM and ethanol, the ninhydrine test was performed to check for the formation of amide bonds. Boc groups were then removed from Phe residues with TFA (25\% in DCM). After filtration and subsequent washing with TEA (1\% in DCM), ethanol $(100 \mathrm{ml})$ and DCM, the ninhydrine test was performed to check for completion of removal of Boc groups. The 
same procedure was repeated until the desired Phe peptide length (Phe ${ }_{4}, \mathrm{CSP} 1 ; \mathrm{Phe}_{8}$, CSP2; Phe 12 , CSP3) was obtained (Fig. 1).

One gram of APS-(Phe) $)_{n}-\mathrm{H}$ was end-capped by reaction with BZ $(140 \mu \mathrm{l})$ in DIEA $(260 \mu \mathrm{l})$ and DCM $(5 \mathrm{ml})$ for $2 \mathrm{~h}$. After performing the ninhydrine test, the reaction mixture, including APS-(Phe $)_{\mathrm{n}}$-BZ, was filtered and washed with DCM and ethanol, then dried in vacuo.

The modification ratios (mmol/g) of Phe peptide on APS was 0.295 (CSP1), 0.202 (CSP2) and 0.155 (CSP3) and were estimated from the value of nitrogen by elemental analysis.

\section{Chromatography}

CSP1-3 products were individually packed into HPLC columns (150 mm x $4.6 \mathrm{~mm}$ i.d.) by a slurry packing method with methanol [17].

The HPLC system included Waters LC Module 1 (Milford, USA), Waters HTR-B column oven and Rikadenki R-01 recorder (Tokyo, Japan). Flow rate was set at 0.5 $\mathrm{ml} / \mathrm{min}$ with $\mathrm{UV}$ detection at $280 \mathrm{~nm}$ and column oven temperature of $35^{\circ} \mathrm{C}$.

All aqueous solutions were made with the water that was deionized and distilled using WG 220 (Yamato Scientific Co, Tokyo) and then passed through a water purification system (Puric-Z, Organo Co, Tokyo). The mobile phase was a mixture of $0.5 \mathrm{M}$ aqueous sodium perchlorate and acetonitrile, adjusted with perchloric acid to $\mathrm{pH}$ 2.0 and was degassed thoroughly prior to use.

The retention factor $(k)$ was determined by $\left(t_{\mathrm{r}}-t_{\mathrm{o}}\right) / t_{\mathrm{o}}$, in which $t_{\mathrm{r}}$ and $t_{\mathrm{o}}$ were retention time of analytes and uracil respectively. Specifically, $k$ values of the first and 
second eluting enantiomers were defined as $k_{\mathrm{A}}$ and $k_{\mathrm{B}}$, respectively. Separation factor $(\alpha)$ was calculated by $k_{\mathrm{B}} / k_{\mathrm{A}}$. Resolution factor $(R s)$ is equal to $1.18 \times\left\{\left(t_{\mathrm{rB}}-t_{\mathrm{rA}}\right) /\right.$ $\left.\left(\mathrm{W}_{1 / 2 \mathrm{~A}}+\mathrm{W}_{1 / 2 \mathrm{~B}}\right)\right\}$, where $t_{\mathrm{rA}}$ and $t_{\mathrm{rB}}$ are the retention times of the first and second eluting enantiomers respectively and $\mathrm{W}_{1 / 2}$ was the peak width at half height.

\section{Infrared spectroscopy}

The CSPs were analyzed by Thermo-Nicolet Nexus 670 FT-IR (32 scans at a resolution of $\left.4 \mathrm{~cm}^{-1}\right)$, using potassium bromide $(\mathrm{KBr})$ pelletization method

\section{Results and discussion}

First, in order to determine the retention mode of CSP1-3, the effect of percentage water $(20-60 \%, v / v)$ in the water-acetonitrile mobile phase on the retention time of methylbenzoate was studied. As water percentage increased, the retention times of the CSPs increased, indicating a reversed-phase retention mode. The order of retention times obtained at each water content was always CSP3 $>$ CSP2 $>$ CSP1, resulting from the CSP with longer Phe peptide having more hydrophobicity and enhancing the retention in reversed-phase mode.

Using five chiral analytes, we evaluated enantioselectivity of the CSPs in HPLC (Table 1). The resolution of warfarin enantiomers was best. Warfarin enantiomers have been widely used as a model chiral analyte in the evaluation of CSPs.

Investigation of the chemical structure of the Phe peptide, reveals that a combination of hydrophobic interaction, $\pi-\pi$ interaction and hydrogen bonding would seem to contribute to the retention mechanism. Accordingly a longer peptide chiral selector 
was expected to be more effective on the chiral separation because of increased hydrophobicity as the Phe peptide length increases and in general, the chiral recognition based on the secondary structure of peptide becomes more pronounced. The parameters $(k, \alpha, R s)$ and the representative chromatograms in the separation of $R / S$-warfarin on CSP1-3, using a mobile phase consisting of $0.5 \mathrm{M}$ aqueous sodium perchlorate $(\mathrm{pH} 2.0)$ and acetonitrile (77/23, v/v), are shown in Table 2 and Fig. 2, respectively. Surprisingly, best resolution was achieved by CSP2, which had an intermediate peptide length. The same tendency was observed for the aminobutyl silica (ABS)-based CSP having the same chiral selectors as CSP1-3 (Table 2).

By making the Phe peptide selectors longer, hydrophobic interaction and $\pi-\pi$ interaction are enhanced. The hydrogen bonding may be decreased, but its influence on the chiral recognition is relatively weak. Therefore, the lower enantioselectivity of CSP3 than that of CSP2 could be attributed to the secondary structure of Phe 12 . The secondary structure of a peptide depends on its chain length [15] and variation in secondary structure could impact on enantioselectivity. An IR spectra is widely used to estimate the conformation of peptides and proteins from their amide I and II absorption bands [12, 13, 18-21]. IR measurement on CSP1-3, initially dispersed in the mobile phase for $12 \mathrm{~h}$ and then dried in vacuo at ambient temperature, was carried out. As shown in Fig. 3, the resultant IR spectra provided an amide I absorption band at $1645-44 \mathrm{~cm}^{-1}$ and an amide II absorption band at $1541-35 \mathrm{~cm}^{-1}$. Absorption bands at $1650-40$ and 1550-20 are characteristic of $\alpha$-helical structure $[18,19]$ and thus the Phe peptides immobilized on CSP1-3 were assumed to be mainly in the $\alpha$-helical state. Therefore the lower enantioselectivity of CSP3 compared to CSP2 could not be 
explained by differences in secondary structure. Further investigation on the reason why CSP2 demonstrated the best enantioselectivity is now in progress.

The better resolution obtained by CSP2 having an intermediate peptide length was in contrast to previous findings where a proline-based CSP showed improved enantioselectivity with longer peptide length [11]. This difference presumably resulted from selection of the amino acid used and thus when developing a peptide chiral selector it would seem to be necessary to study the effect of peptide length, rather than relying on theoretical principles.

Using a second lot (lot 2) of APS, we prepared CSP2 for evaluation of reproducible preparation. The immobilization ratio of lot $2(0.221 \mathrm{mmol} / \mathrm{g})$ was larger than that of lot $1(0.202 \mathrm{mmol} / \mathrm{g})$, which was likely to be due to the higher presence of aminopropyl groups on the silica in lot 2 (lot $1,0.700 \mathrm{mmol} / \mathrm{g}$; lot $2,0.793 \mathrm{mmol} / \mathrm{g}$ ). Resolution of $R / S$-warfarin was almost equivalent (lot 1, 1.60; lot 2, 1.64) using the same HPLC conditions.

Finally, in order to study the effect of end-capping groups in CSP2 on the chiral separation, three CSP2 end-capped by BZ, CH and DNB (i.e., APS-(Phe) ${ }_{8}$-BZ, APS-(Phe $)_{8}-\mathrm{CH}$ and APS-(Phe) $\left.)_{8}-\mathrm{DNB}\right)$ and non end-capped CSP2 (i.e., APS-(Phe) $\left.{ }_{8}-\mathrm{H}\right)$ were evaluted (Table 3). The non-enantioselectivity of APS-(Phe) $)_{8}-\mathrm{H}$ clearly demonstrated the importance of an end-capping reagent. Althogh APS-(Phe) ${ }_{8}-\mathrm{CH}$ is more hydrophobic than APS-(Phe) ${ }_{8}-\mathrm{BZ}(\log \mathrm{P}:-\mathrm{CH}, 3.39$; -BZ, 2.22 [22]), it was expected that the $\pi$-acidic moiety at the $\mathrm{N}$-terminus of APS-(Phe) $)_{8}-\mathrm{BZ}$ would facilitate enantioselectivity by enhanced $\pi-\pi$ interaction. However, APS-(Phe) ${ }_{8}$-DNB was not so good as expected and APS-(Phe $)_{8}-\mathrm{CH}$ was better, which was likely to be due to the 
lower hydrophobicity of the former. These results suggest that $\pi-\pi$ and hydrophobic interactions between the end-capped groups and analytes coordinately contributed to the enantioselectivity.

In summary, a new class of CSPs with a different number of Phe units immobilized on APS have been prepared and evaluated. CSP3 with the longest Phe peptide selector did not demonstrate the best enantioselectivity as expected, but the highest resolution was achieved by the CSP2 having intermediate peptide length. This indicated that enhanced enantioselectivity is not always obtained by a longer peptide chiral selector. Therefore, when a different amino acid is used to develop a peptide chiral selector, optimization of peptide length can enhance enantioselectivity.

\section{Acknowledgement}

The authors thank Daiso co., LTD. (Osaka) for the kind gift of 3-aminopropyl silyl silica gel. The authors thank Drs. G.A Lord (London University) and Y. Sun (Shanghai Institute of Materia Medica) for their kind suggestion. 


\section{References}

[1] I.W. Muderawan, T.-T. Ong, S.-C. Ng, J. Sep. Sci. 29 (2006) 1849.

[2] A. Berthod, X. Chen, J.P. Kullman, D.W. Armstrong, F. Gasparrini, I. D’Acquarica,

C. Villani, A. Carotti, Anal. Chem 72 (2000) 1767.

[3] E. Yashima, C. Yamamoto, Y. Okamoto, J. Am. Chem. Soc. 118 (1996) 4036.

[4] W.H. Pirkle, J.M. Finn, J.L. Schreiner, B.C. Hamper, J. Am. Chem. Soc. 103 (1981) 3964.

[5] C.J. Welsh, J. Chromatogr. A 666 (1996) 3.

[6] G. Schill, I.W. Wainer, S.A. Barkan, J. Chromatogr. 365 (1986) 73.

[7] M. Lämmerhofer, W. Lindner, J. Chromatogr. A 741 (1996) 33.

[8] T. Li, J. Sep. Sci. 28 (2005) 1927.

[9] A. Dobashi, Y. Dobashi, K. Kinoshita, S. Hara, Anal. Chem. 60 (1988) 1985.

[10] J. Huang, P. Zhang, H. Chen, T. Li, Anal. Chem. 77 (2005) 3301.

[11] J. Huang, H. Chen, T. Li, J. Chromatogr. A 1113 (2006) 109.

[12] A. Shundo, T. Sakurai, M. Takafuji, S. Nagaoka, H. Ihara, J. Chromatogr. A 1073 (2005) 169.

[13] Y.Chen, D.-H. Yu, J. App. Poly. Sci. 49 (1993) 851.

[14] S.G. Allenmark, S. Andersson, J. Chromatogr. A 666 (1994) 167.

[15] T.E Creighton, Proteins: Structures and Molecular Properties, W.H. Freeman and Co., New York, 1993.

[16] B.D. West, S. Preis, C.H. Schroeder, K.P. Link, J. Am. Chem. Soc. 83 (1961) 2676.

[17] H. Guan-Sajonz, G. Guiochon, J. Chromatogr. A 743 (1996) 247.

[18] T. Miyazawa, E.R. Blout, J. Am. Chem. Soc. 83 (1961) 712.

[19] H. Hiramatsu, T. Kitagawa, Biochim. Biophys. Acta 1753 (2005) 100. 
[20] A. Dong, P. Huang, W.S. Caughey, Biochemistry 29 (1990) 3303.

[21] A. Mauerer, G. Lee, Eur. J. Pharm. Biopharm. 62 (2006) 131.

[22] SciFinder Scholar, The values Calculated Using Advanced Chemistry Development Software Solaris, American Chemical Society, Washington, DC, 2006. 


\section{Figure captions}

Fig. 1 Structure of poly(L-phenylalanine) chiral stationary phase.

Fig. 2 Typical chromatographic separations of $R / S$-warfarin with CSP1-3. Mobile phase: $0.5 \mathrm{M}$ sodium perchlorate $(\mathrm{pH} 2.0)$-acetonitrile $(77 / 23, \mathrm{v} / \mathrm{v})$. Other conditions as mentioned in Experimental section.

Fig. 3 FT-IR spectra of APS and CSP1-3 after dispersion in the mobile phase for $12 \mathrm{~h}$ and dryness in vacuo. 
Table 1 Chromatographic resolution of chiral analytes with the CSPs

\begin{tabular}{lcccc}
\hline \multicolumn{1}{c}{ chiral analyte } & $k_{\mathrm{A}}$ & $k_{\mathrm{B}}$ & $\alpha$ & $R s$ \\
\hline Trihexyphenidyl $^{\mathrm{a})}$ & 20.7 & 21.3 & 1.03 & 0.955 \\
Warfarin $^{\mathrm{b})}$ & 25.6 & 26.9 & 1.05 & 1.31 \\
Ketoprofen $^{\mathrm{b})}$ & 19.0 & 19.0 & 1.00 & 0 \\
Chloropheniramine $^{\mathrm{c})}$ & 9.86 & 9.86 & 1.00 & 0 \\
Benzoin $^{\mathrm{d})}$ & 20.6 & 21.3 & 1.03 & 1.01 \\
\hline
\end{tabular}

a) CSP3, 0.5M sodium perchlorate $(\mathrm{pH} 2.0) /$ Acetonitrile $=70 / 30(\mathrm{v} / \mathrm{v})$

b) CSP3, 0.5M sodium perchlorate $(\mathrm{pH} 2.0) /$ Acetonitrile $=75 / 25(\mathrm{v} / \mathrm{v})$

c) CSP3, 0.5M sodium perchlorate $(\mathrm{pH} 2.0) /$ Acetonitrile $=85 / 15(\mathrm{v} / \mathrm{v})$

d) CSP2, 0.5M sodium perchlorate $(\mathrm{pH} 2.0) /$ Acetonitrile $=85 / 15(\mathrm{v} / \mathrm{v})$ 
Table 2 Chiral separation of $R / S$-wafarin on the CSP1-3

\begin{tabular}{|c|c|c|c|c|c|}
\hline & & $k_{\mathrm{A}}$ & $k_{\mathrm{B}}$ & $\alpha$ & $R s$ \\
\hline \multirow[t]{3}{*}{ APS-(Phe)n-BZ } & $\mathrm{n}=4 \quad(\mathrm{CSP} 1)$ & 20.1 & 20.8 & 1.04 & 1.03 \\
\hline & $\mathrm{n}=8 \quad(\mathrm{CSP} 2)$ & 27.3 & 29.2 & 1.07 & 1.60 \\
\hline & $\mathrm{n}=12(\mathrm{CSP} 3)$ & 31.0 & 32.7 & 1.05 & 1.31 \\
\hline \multirow[t]{3}{*}{ ABS-(Phe)n-BZ } & $\mathrm{n}=4$ & 21.3 & 23.1 & 1.04 & 0.74 \\
\hline & $\mathrm{n}=8$ & 30.1 & 32.3 & 1.07 & 1.48 \\
\hline & $\mathrm{n}=12$ & 46.4 & 48.7 & 1.05 & 0.99 \\
\hline
\end{tabular}


Table 3 Effect of end-capping reagents on the enantioselectivity

\begin{tabular}{lcc}
\hline & $\alpha$ & $R s$ \\
\hline APS-(Phe)8-BZ* & 1.07 & 1.64 \\
APS-(Phe)8-DNB* & 1.05 & 0.99 \\
APS-(Phe)8-CH* & 1.06 & 1.30 \\
APS-(Phe)8-H* & 1.00 & 0 \\
\hline
\end{tabular}

* made of APS (lot 2) 
Fig. 1

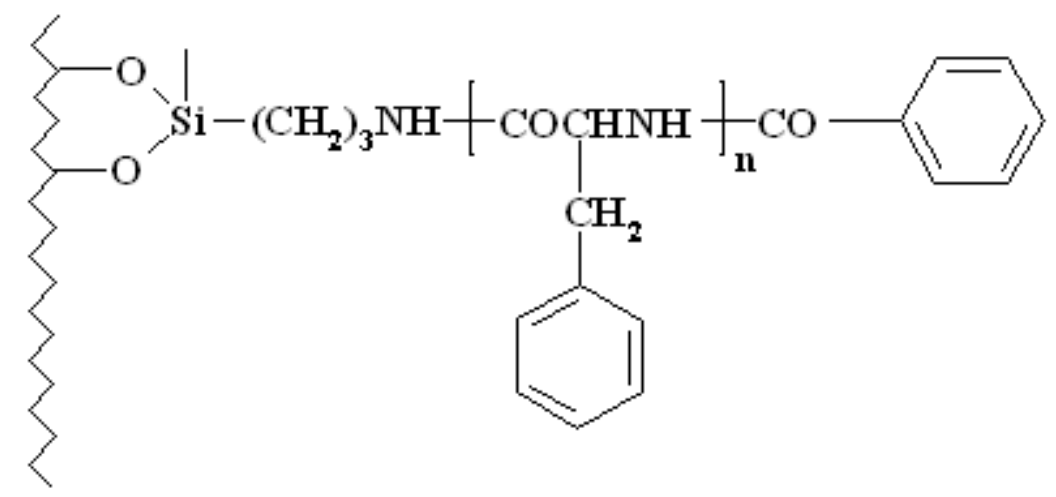

K. Ohyama et al. 
Fig. 2
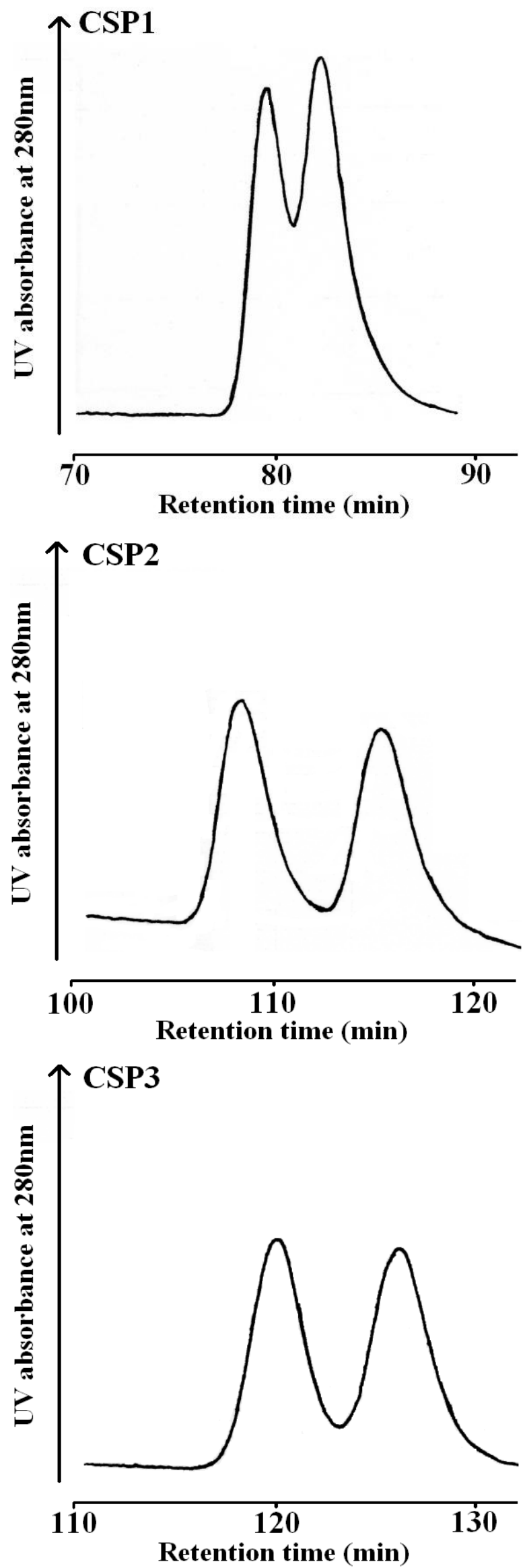

K. Ohyama et al. 
Fig. 3

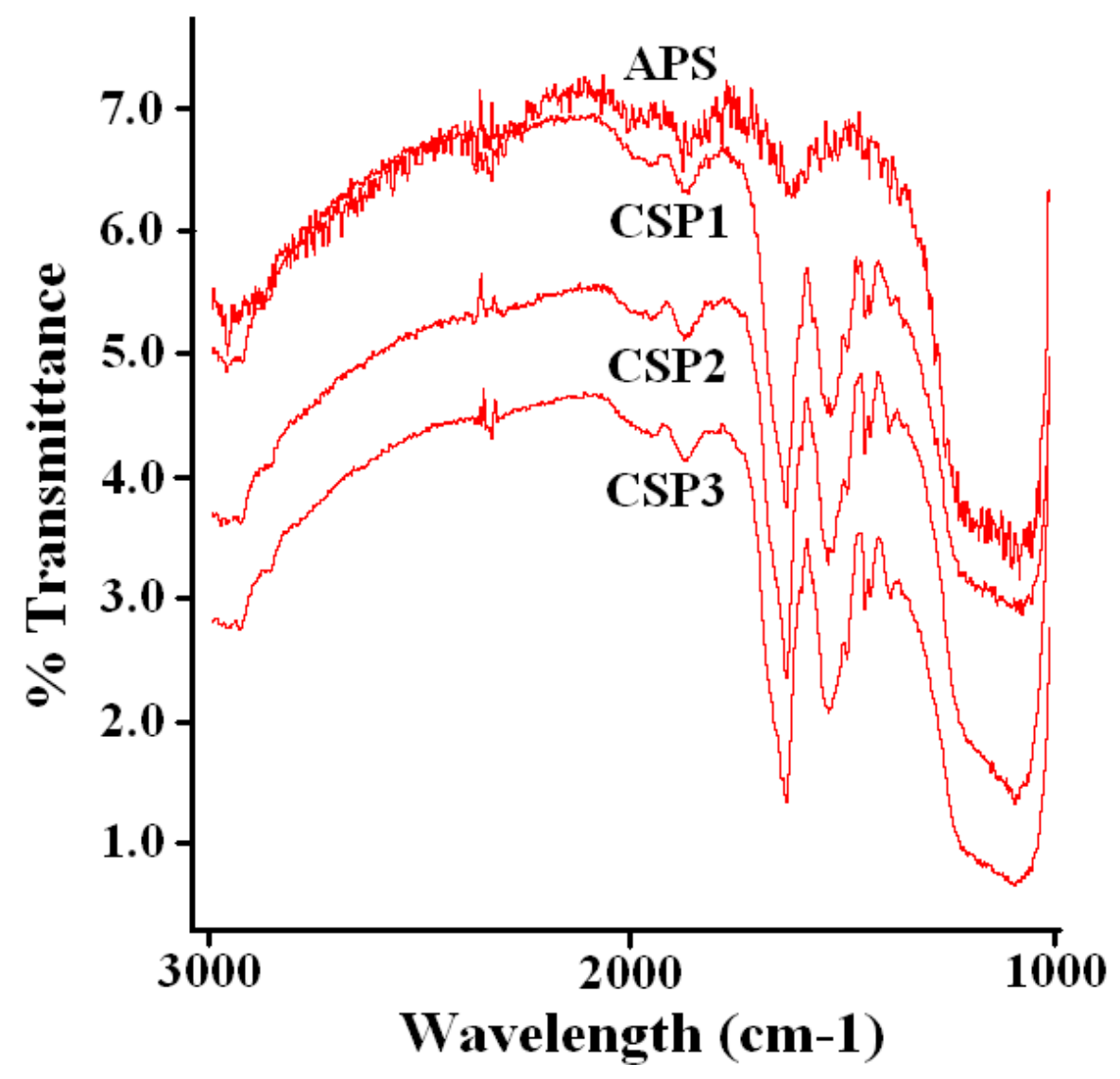

K. Ohyama et al. 